\title{
Structure for Ultrasonic Suspension Gap Pressure Sensor
}

\author{
Masaya Takasaki, ${ }^{*}$ Ryutaro Chida, Shota Chino, Satoshi Morishita, ${ }^{1}$ \\ Yuji Ishino, Kota Hosaka, ${ }^{1}$ Yoshio Mita, ${ }^{1}$ and Takeshi Mizuno \\ Saitama University, 255 Shimo-Okubo, Sakura-ku, Saitama 338-8570, Japan \\ ${ }^{1}$ The University of Tokyo, 7-3-1 Hongo, Bunkyo-ku, 113-8654, Japan
}

(Received September 21, 2016; accepted January 4, 2017)

Keywords: pressure sensor, diaphragm, MEMS, ultrasonic vibration, non-contact suspension

It has been reported that an object can be suspended below a vibrating surface in air. We call this phenomenon ultrasonic suspension. When an object is suspended, a restoring force attracts the object horizontally to the center of the vibrating surface. Previously, characteristics of the forces acting vertically and horizontally were experimentally investigated using a servo-type measuring mechanism. While ultrasonic vibration was being excited, actuation forces acting on the object were measured. This characterization implied that negative pressure is generated in the suspension gap. To determine the principles governing these forces, the pressure distribution in the gap was investigated. Two types of pressure sensors were prepared. Measurements by these sensors did not agree with the characterized force, because a small hole at the measuring point in both sensors influenced the results. Therefore, we proposed a novel pressure sensor employing a diaphragm. Deflection of the diaphragm in proportion to applied pressure can be expected. The sensor was prototyped by micro-electromechanical system (MEMS) technology and calibrated. Results show that the deflection was proportional to the applied pressure and had no hysteresis.

\section{Introduction}

Recently, a non-contact handling technique that uses the ultrasonic levitation phenomenon as a means of transporting semiconductor substrates or liquid crystal display (LCD) glass substrates has been considered. ${ }^{(1-4)}$ Specifically, the near-field levitation phenomenon has been observed. Near-field levitation is the phenomenon in which a planar object can be levitated upward above a vibrating surface with a gap of tens of micrometers in air, as shown in Fig. 1(a). Theoretical analysis considering air viscosity has revealed that a force acting on a circular plate reverses from repulsive to attractive with increasing levitation distance in the case of near-field levitation. ${ }^{(5)}$

In contrast to ultrasonic levitation, a phenomenon in which a planar object can be suspended under a vibrating surface without contact underwater ${ }^{(6)}$ and in air, ${ }^{(7)}$ as illustrated in Fig. 1(b), has been experimentally demonstrated. In the case of suspension in air, the suspension gap was a few tens to $100 \mu \mathrm{m}$. This phenomenon is called ultrasonic suspension and induces both vertical and horizontal actuation forces. The vertical force balances gravity acting on the object. The horizontal force attracts the object to the center of the vibrating surface when there is horizontal misalignment. It acts as a restoring force. Because of these actuation forces, a suspended object can be held passively without active control.

*Corresponding author: e-mail: masaya@ieee.org http://dx.doi.org/10.18494/SAM.2017.1471 


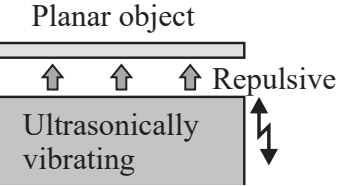

(a)

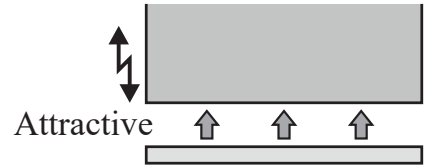

(b)

Fig. 1. Ultrasonic levitation and ultrasonic suspension. (a) Levitation. (b) Suspension.

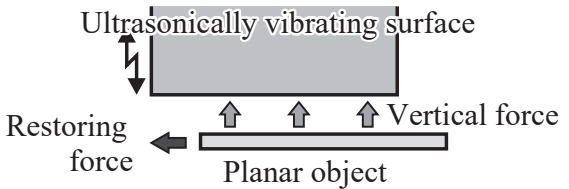

Fig. 2. Horizontal actuation force.

Previously, the actuation forces were characterized with a servo-type force measuring mechanism. ${ }^{(9)}$ The vertical force and the restoring force act on the object as illustrated in Fig. 2. The forces were measured as a function of changes in vibration amplitude, suspension gap, object thickness, and center distance. In the case of vertical direction, i.e., the force acting on the object perpendicular to the surface facing the ultrasonically vibrating surface through the gap, the force was attractive for a wider gap and repulsive for a smaller gap.

It was also observed that the attractive force was not induced in the case of a suspended object whose diameter was larger than $8 \mathrm{~mm} .{ }^{(9)}$ To apply this phenomenon to a non-contact handling technique, tests of suspension for larger targets were carried out. ${ }^{(10)}$ In the tests, the vibrating surface was divided into segments. An attractive force was observed when the diameter of the vibrating surface was as large as $30 \mathrm{~mm}$.

According to the results of the characterization of the actuation forces, it seems that negative pressure is induced in the suspension gap when the force is attractive. Our interest then focused on the principles of the negative pressure. Negative squeeze ${ }^{(11)}$ is one of the possibilities. To examine the pressure principle, the distribution of pressure in the gap was measured. Two types of pressure sensors for measuring local pressure in the gap were tested. The results, however, did not agree with experimentally characterized forces. We then proposed and designed a novel pressure sensor for the measurement. The sensor was prototyped with micro-electromechanical system (MEMS) technology and calibrated with an experimental apparatus. Trial measurements of suspension gap pressure were carried out.

The remaining part of this paper is organized as follows. Ultrasonic suspension is introduced in Sect. 2. An example of the characterization results is included. Trial measurements of pressure distribution in the gap with two types of sensors are reported in Sect. 3. A proposal for a novel pressure sensor is presented in Sect. 4. Fabrication, calibration, and trial measurements are also reported. The final section contains our conclusions.

\section{Ultrasonic Suspension}

An object can be levitated vertically upward above a vibrating surface of an ultrasonic transducer as shown in Fig. 1(a) if the intensity of the vibration is strong enough. On the other hand, it has been reported that an object can be suspended below a vibrating surface in air, ${ }^{(8)}$ as illustrated in Fig. 1(b). We call this phenomenon ultrasonic suspension. The suspension can be applied to the handling of a small object, since the object is pulled to the center of the vibrating surface when it is initially misaligned. This implies that a horizontal actuation force as well as a vertical actuation force acts on the object, as illustrated in Fig. 2. 
These forces were experimentally characterized with a servo-type force measurement system. ${ }^{(9)}$ The actuation forces in the vertical and horizontal directions during ultrasonic suspension were measured by the setup schematically illustrated in Fig. 3. The target object was fixed on a base, which was suspended by two piano-wire rods to provide three degrees of freedom to the object. Position and orientation of the base were measured by sensors and controlled by three voice coil motors. Applying proportional-integral-differential control, the base can be in an original position and orientation while the actuation force acts on the object. As a result, the actuation force can be estimated from coil currents. An ultrasonic transducer with a horn was placed horizontally to cancel gravity acting on the target object. To adjust the diameter of the vibrating surface, a cap was fixed on the top of the horn with a built-in screw. By replacing the cap, the diameter of the vibrating surface can be changed. During the experiment, the resonance frequency of the ultrasonic transducer was tracked by a resonance frequency tracking system ${ }^{(12)}$ to maintain a large vibration amplitude in the transducer. The frequency was $28 \mathrm{kHz}$.

An example of the characterized force under conditions of a vibration amplitude of $2.0 \mu \mathrm{m}_{\mathrm{p}-\mathrm{p}}$, an object diameter of $6 \mathrm{~mm}$, and a vibrating surface diameter of $6 \mathrm{~mm}$ is shown in Fig. 4. For a smaller gap, the force was repulsive and agreed with the squeeze film effect. In contrast, the direction of the force was reversed for wider gaps and was attractive. Close to the reversing point, the force is that of positive stiffness. This means that the suspended object can be held where the force balances gravity acting on the object. Additionally, air around the suspended object has a damping effect. Consequently, these properties resulted in a passively stable suspension.

\section{Measurement of Pressure Distribution in the Gap}

According to properties of an actuation force during ultrasonic suspension, the average pressure in the gap is negative; namely, the absolute value of the local pressure at a certain point in the gap is lower than that of the atmosphere. To discuss the principles of the negative pressure in the gap,

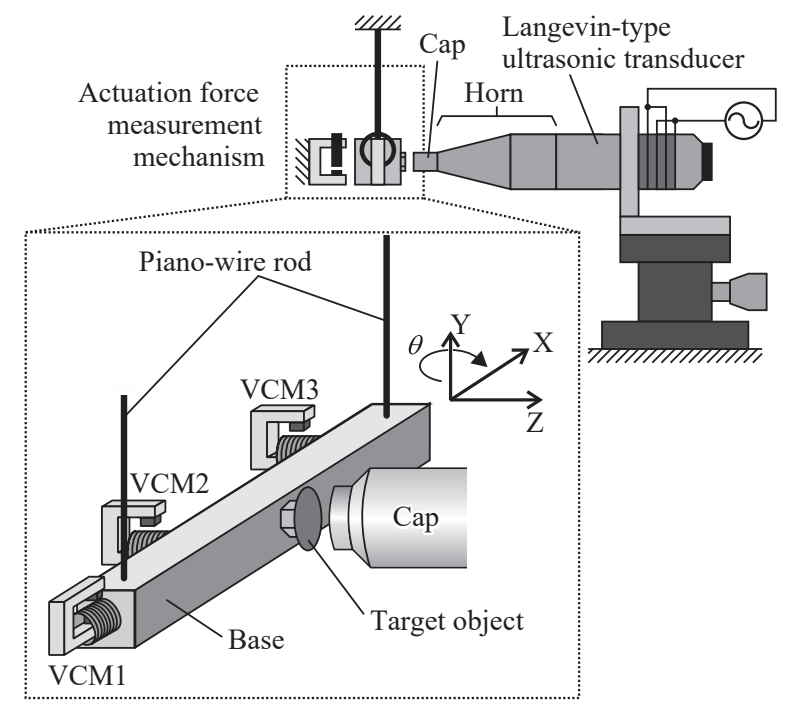

Fig. 3. Servo-type actuation force measuring system.

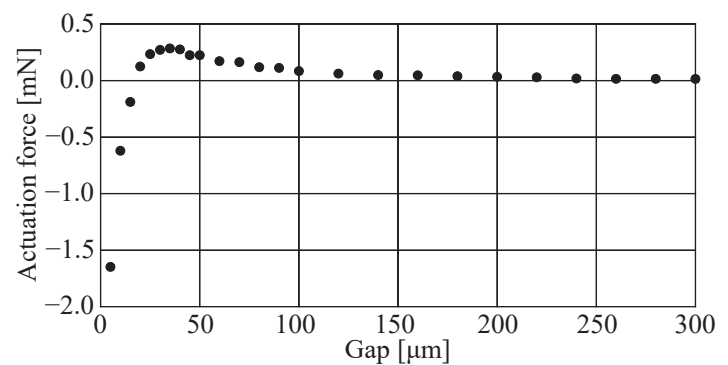

Fig. 4. Characterized actuation force as a function of gap size. (Vibration amplitude $=2 \mu \mathrm{m}_{\mathrm{p}-\mathrm{p}}$ ). 
we have focused on investigating the pressure distribution in the gap. We expected the distribution of positive pressure in the center to be due to the squeeze film effect and the negative pressure in the circumference to be induced by the negative squeeze effect. Observing the precise distribution would support a deep understanding of the principles. In this research, the term "pressure" means time-averaged air pressure, not sound pressure.

To investigate the distribution, the experimental setup schematically illustrated in Fig. 5 was used. A Langevin-type ultrasonic transducer whose resonant frequency was $28 \mathrm{kHz}$ was connected to a horn and fixed vertically. Then, a pressure sensor was positioned to face the vibrating surface on the top of the horn. The relative position of the sensor was adjusted by a stage to which the sensor was attached. To simulate ultrasonic suspension, the sensor surface was configured with a circular plate. To investigate the pressure distribution, two types of pressure sensors were prepared as indicated in the figure.

The first type employed a general pressure sensor whose differential pressure range was from 0 to $200 \mathrm{~Pa}$. The circular plate had a hole, whose diameter was $0.3 \mathrm{~mm}$, connected to the sensor. The connection was realized with a rubber tube. The second type consisted of a piezo-resistancetype pressure sensor, whose range of absolute pressure was from 50 to $110 \mathrm{kPa}$. The sensor was embedded on a printed circuit board for wiring. The sensor was fixed in the plate as shown in the figure. A small hole $0.15 \mathrm{~mm}$ in diameter was located on the top surface of the sensor.

The pressure distribution as measured by the first sensor is plotted in Fig. 6 as a function of the size of gap $G$. The vibration amplitude was fixed at $2 \mu \mathrm{m}_{\mathrm{p}-\mathrm{p}}$ during the measurement. The pressure in the gap was positive at every position and gap size. This means the average pressure is positive and does not induce an attractive force during ultrasonic suspension, although an attractive force acted at the gap of $60 \mu \mathrm{m}$ in Fig. 4 .

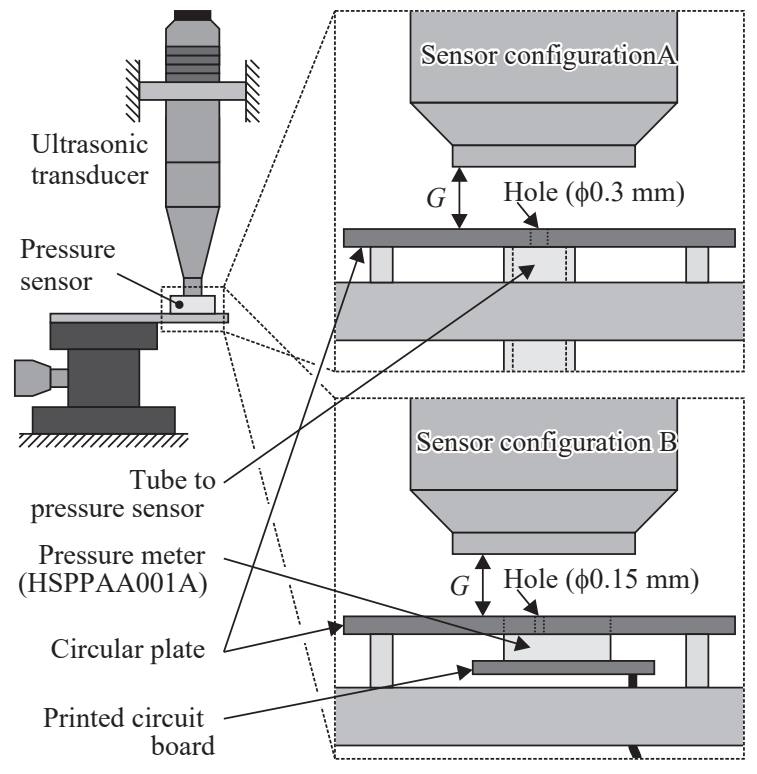

Fig. 5. Pressure sensor configurations to investigate pressure distribution in the suspension gap.

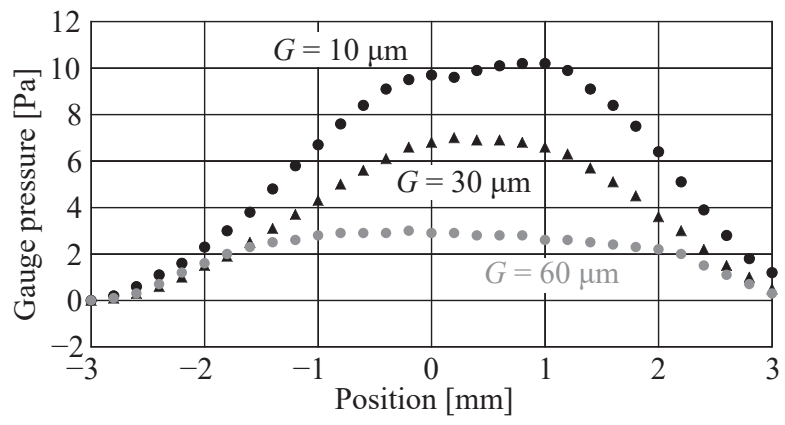

Fig. 6. Pressure distribution in the suspension gap as measured by a pressure sensor connected through a tube. 
The second type of pressure sensor was also applied to measure the distribution for a vibration amplitude of $2 \mu \mathrm{m}_{\mathrm{p}-\mathrm{p}}$. The observed pressure distribution is plotted in Fig. 7. The tendency was opposite to that measured by the first type. The pressure was negative at every position even with a gap size of $20 \mu \mathrm{m}$. In this case, the actuation force estimated from this result does not agree with the properties characterized by the force measurement system. Additionally, the direction of the total force as the integral of overall results is opposite to that shown in Fig. 6.

The ultrasonic suspension actuating forces estimated from the measured pressure distributions did not agree with those characterized previously. It seems that air flow around the sensor hole influenced the measurements. The negative squeeze effect may influence the negative pressure principle as mentioned. This effect is induced at the edge of a vibrating surface during ultrasonic suspension as illustrated on the right-hand side of Fig. 8. Microscopically, the same situation exists around the sensor holes as shown on the left-hand side of the figure and induces the same effect around the holes. Both sensor structures had holes on their surfaces. On the other hand, the volume behind each hole and the diameter of each hole are different. These situations imply that air flow around the hole influenced the average pressure sensing element of each sensor. Therefore, a hole in the structure of a pressure sensor is not suitable for detection of the local pressure in the suspension gap. Consequently, we have proposed a novel structure for a pressure sensor for an ultrasonic suspension gap.

\section{Novel Pressure Sensor}

\subsection{Concept}

To investigate the pressure distribution under the same condition as an ultrasonic suspension, a pressure sensor without a hole is required, because the hole may induce error in the value of measured local pressure. Therefore, we proposed a novel pressure sensor suitable for measuring local pressure in a suspension gap. The proposed sensor was configured without the hole.

In our proposal, the pressure sensor has a diaphragm as its micro-structure; the concept is depicted in Fig. 9. Basically, the sensor is a substrate, whose diameter is the same as that of the suspended object or larger than the vibrating surface of the ultrasonic transducer; it can simulate the suspended object. The sensor surface faces the top of the ultrasonic transducer and includes

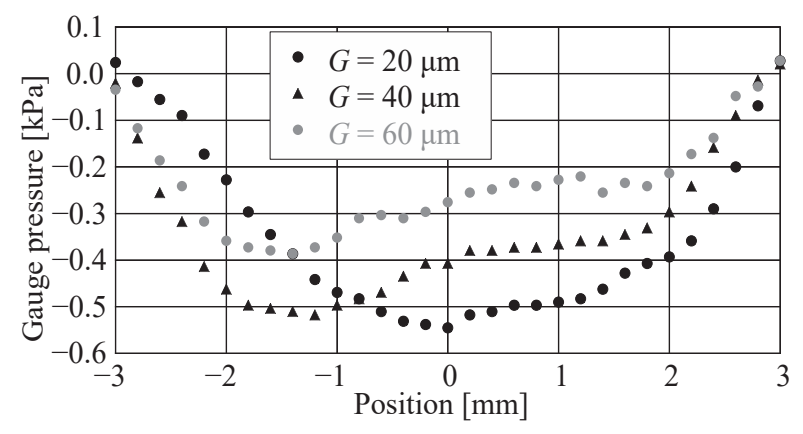

Fig. 7. Pressure distribution in the suspension gap as measured by a pressure sensor embedded in the circular plate.

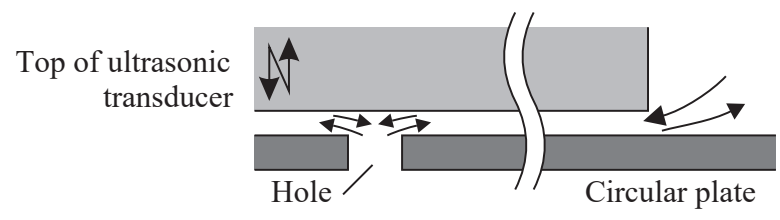

Fig. 8. Air flow around the edge of a vibrating surface and sensor hole. 


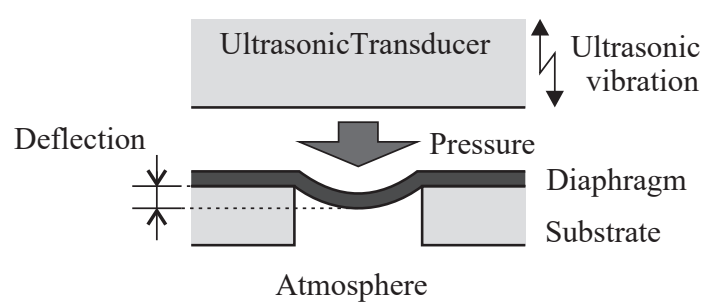

Fig. 9. Concept of the proposed sensor.

the diaphragm. When an ultrasonic vibration is generated, the diaphragm can be deformed by the pressure induced in the gap, as illustrated in the figure. If the vertical displacement of the center of the diaphragm can be monitored as the diaphragm deflection, local pressure behind the diaphragm can be estimated from the deflection.

Applying MEMS technology, a micro-structure can be simultaneously fabricated. Therefore, the proposed structure can be arrayed as illustrated in Fig. 10. Because of the array, the pressure distribution in the gap can be captured in a one-time measurement. To realize the measurement, independent measurement of the deflection of each diaphragm is required. Figure 11 depicts the possibilities for independent measurement. (a) An electrode attached on the diaphragm and a reference electrode can configure a capacitor. Diaphragm deflection can be estimated by the change in its capacitance. (b) The piezoresistance equipped on the back of the diaphragm depends on the bending deformation of the diaphragm. By measuring the resistance, the deformation can be calculated. Within these possibilities, the value of capacitance or resistance should be detected. Therefore, an analogue signal processing circuit is needed for detection. The processing circuit can be integrated on the same substrate as the sensor, because the sensor structure is embedded on/in a silicon wafer as a result of the MEMS technology.

\subsection{Prototype}

Considering the practical fabrication of a sensor based on this concept, a silicon wafer was selected as the substrate. The surface of the substrate was polished to simulate the suspended object even if it is coated. A polyimide film was considered for the diaphragm, because the film can be prepared by spin coating on a wafer. To realize the micro-scale diaphragm structure, the wafer can be etched. The diaphragm deflection detecting mechanism was not included in this prototype, because this research is a feasibility study of the concept. The deflection could be detected in an alternative way such as with a laser displacement sensor. A prototype with this sensor structure was fabricated by the processes shown in Fig. 12.

The detailed fabrication process is as follows. (a) Both sides of the silicon wafer were coated with aluminum film by evaporation. Then, a polyimide film was prepared on the top surfaces by a spin coating and baking. The bottom side of the wafer was covered by a negative photoresist layer. (b) The photoresist layer was exposed through a photomask, then developed. (c) The aluminum film outside the remaining photoresist was etched. (d) The naked part of the silicon wafer was etched by reactive ion etching. Aluminum does not react with reactive ions. During etching, the remaining aluminum film on the bottom surface protected the remaining silicon wafer. On the other hand, the aluminum film on the top surface protected the polyimide layer under the film. (e) The fabrication process was completed with the removal of the photoresist on the bottom surface. 


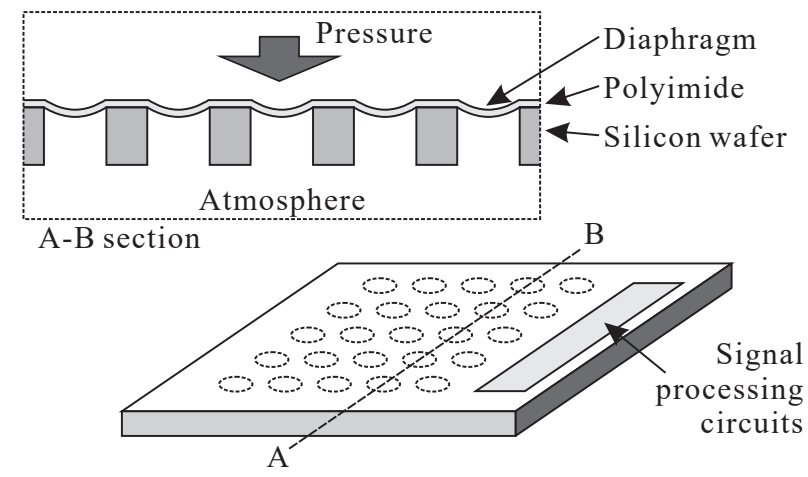

Fig. 10. Concept of the sensor array.

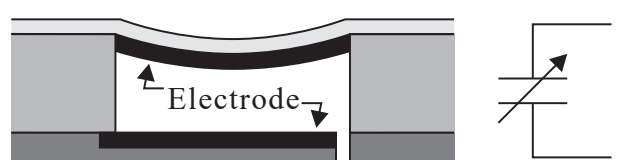

Fig. 11. Diaphragm deformation sensing methods.

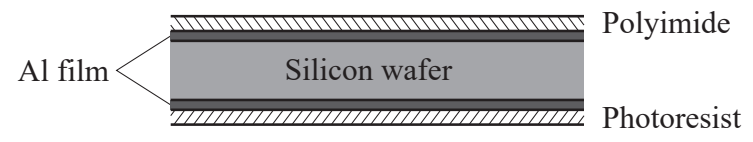

(a) Metal evaporation on both sides, then coating of the photoresist and polyimide

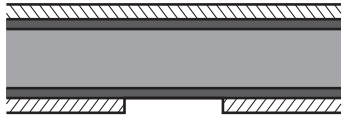

(b) Exposure and development

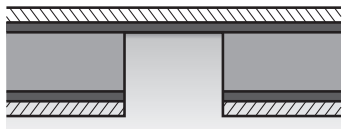

Plasma

(d) Reactive ion etching

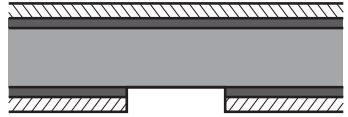

(c) Etching

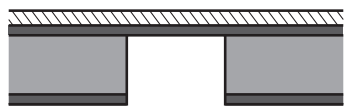

(e) Removing photoresist

Fig. 12. Fabrication of proposed pressure sensor.

The aluminum film remaining behind on the top polyimide layer reflected the laser beam from the displacement sensor.

Two prototypes were fabricated. One type had a single diaphragm, as shown in Fig. 13(a). To simplify positioning the diaphragm, two markers were located on the line including the diaphragm. This type is called Type A. Another type has an array of diaphragm sensors, as shown in Fig. 13(b). This prototype is called Type B. The diaphragms were square. The size of the square is denoted by $L$ in the figure and varied from 400 to $800 \mu \mathrm{m}$ in increments of $100 \mu \mathrm{m}$. The interval on diaphragms $P$ for Type B was $2 L$. Therefore, 5 sensors were prototyped for each type. The atmosphere side of the prototypes and polyimide side, namely, the measurement side, are shown in Fig. 14. For convenience in fabrication, the sensors were fabricated on a 4-inch silicon wafer $525 \mu \mathrm{m}$ thick. The wafer was cut into pieces with sensor structures and markers, as seen in the photograph.

\subsection{Calibration}

For feasibility tests, the prototyped sensors were calibrated with the experimental apparatus shown in Fig. 15. The prototyped sensor was sandwiched by a base and a pressuring block. The 


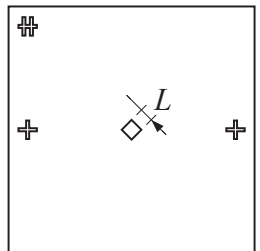

(a)

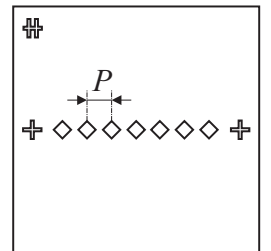

(b)

Fig. 13. Shapes of sensor diaphragms. (a) Type A and (b) Type B.

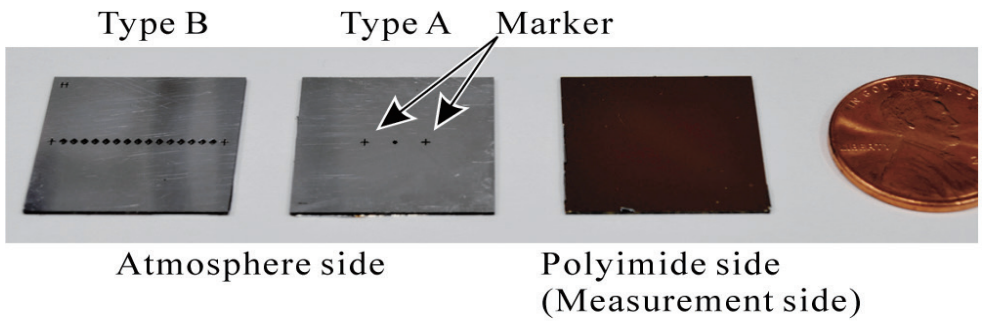

Fig. 14. (color online) Fabricated prototype pressure sensors.

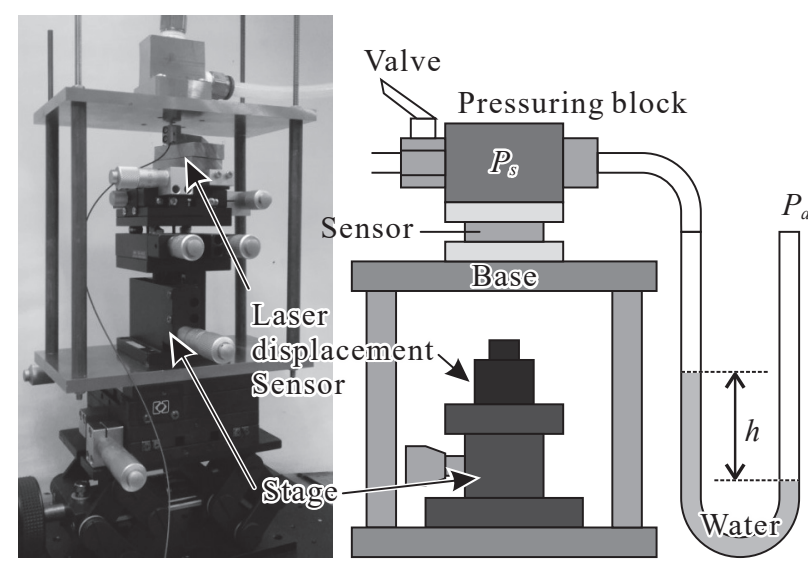

Fig. 15. Pressure sensor calibration setup.

polyimide side faced upward. By providing pressurized/decompressed air at pressure $P_{S}$ to the block, the polyimide side can be pressurized or decompressed. Deflection measurements were carried out while the valve that provided air was closed. Accurate pressure behind the diaphragm was measured by a manometer as illustrated in the figure. The opposite end of the manometer was connected to the atmosphere, whose pressure was $P_{a}$. The pressure can be calculated by the following equation.

$$
P_{a}-P_{s}=\rho g h,
$$

where $h, \rho$, and $g$ are the height difference in the manometer, the density of water, and gravity, respectively. Displacement of the diaphragm was measured by a laser displacement sensor (KEYENCE SI-F).

Type A with $L=800 \mu \mathrm{m}$ was calibrated with the apparatus. The pressure was increased then decreased during the calibration. Diaphragm deformation as a function of applied pressure is 
plotted in Fig. 16. Regarding the sign of the vertical axis, an upward displacement is positive. The deflection of the diaphragm is linear in the range of -5 to $5 \mathrm{kPa}$. Its sensitivity as a pressure sensor was $2.6 \mu \mathrm{m} / \mathrm{kPa}$. Hysteresis was not observed.

Five prototypes of Type B were also calibrated with the apparatus. The center diaphragm of each prototype was tested. Calibration results as a function of diaphragm size are plotted in Fig. 17. This type is linear over the same range as Type A. Focusing on sensitivity, the larger the diaphragm size, the higher its sensitivity.

\subsection{Trial measurement}

Trial measurements of the pressure in the gap were carried out. The calibrated sensor diaphragm $(L$ $=700 \mu \mathrm{m})$ faced the vibrating surface with $6.4 \mu \mathrm{m}_{\mathrm{p}-\mathrm{p}}$ amplitude. Sensor outputs as a function of the gap size are plotted in Fig. 18. The output value jumped suddenly at a gap of $120 \mu \mathrm{m}$. As the gap was decreased, values often jumped. Correct measurement was not possible due to these jumps. To investigate the reason for the jump in values, the vibration of the diaphragm was measured under conditions of suspension. As a result, an ultrasonic vibration whose frequency was the same as the operating frequency of the ultrasonic transducer was observed for a gap of

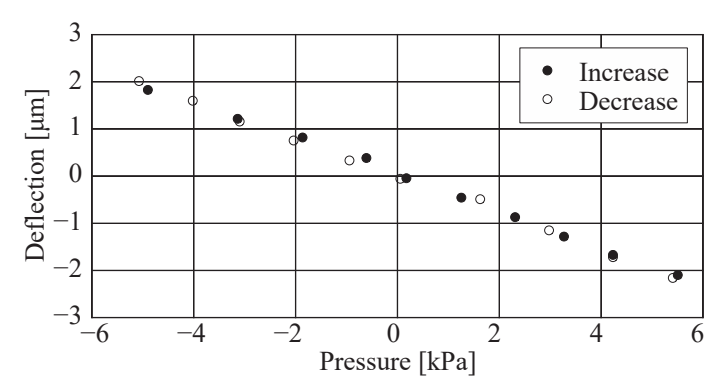

Fig. 16. Calibration result for Type A $(L=800 \mu \mathrm{m})$.

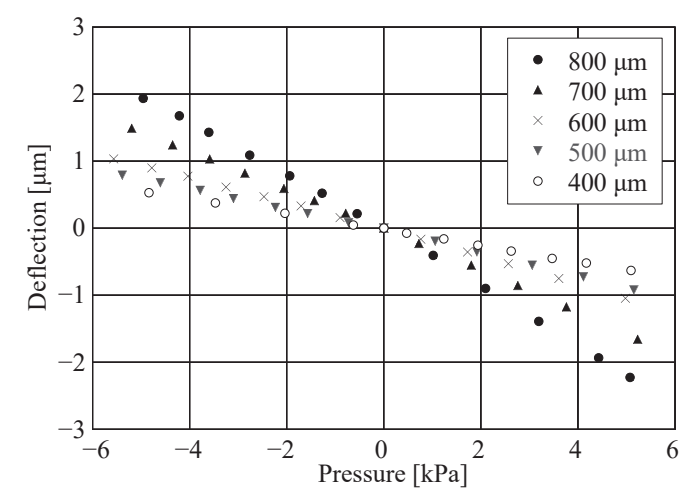

Fig. 17. Calibration results of Type B as a function of diaphragm size.

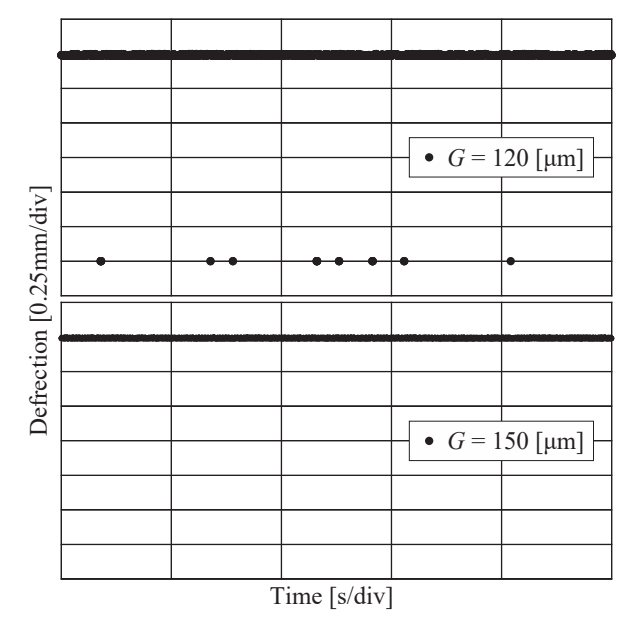

Fig. 18. Output of displacement sensor. 
$200 \mu \mathrm{m}$ and an amplitude of $2 \mu \mathrm{m}_{\mathrm{p}-\mathrm{p}}$. It seems that the diaphragm vibration induced the jump in the output value of the sensor. To realize correct measurement, the diaphragm vibration excited by the ultrasonic vibration should be suppressed. One possibility of the vibration suppression may be adding a weight on the diaphragm. The added weight can decrease the response of the diaphragm against external vibrations. To realize the diaphragm structure with the weight, the structure design and fabrication process of the proposed sensor should be improved.

\section{Conclusion}

To investigate pressure distribution in ultrasonic suspension, two types of pressure sensors were prepared and the conditions of suspension were simulated. Local pressures in the gap were recorded and compiled to evaluate the pressure distribution. These results did not agree with previously characterized actuation forces during ultrasonic suspension. The reason for the disagreement may be the influence of the holes connecting the measuring point and the sensor. To investigate the distribution, the authors proposed a novel pressure sensor, which has a flat surface facing the measuring point. Two prototypes were fabricated with MEMS technology. The sensors were calibrated with an experimental apparatus. Their sensitivity as pressure sensors was 2.6 $\mu \mathrm{m} / \mathrm{kPa}$. With the developed sensor, trial measurements were carried out. Correct values could not be obtained due to ultrasonic vibration of the diaphragm. Improvement of the pressure sensor to suppress the diaphragm vibration will be investigated next. Finally, pressure distribution in the gap during ultrasonic suspension will be able to be observed by an improved sensor.

\section{Acknowledgments}

The MEMS device was fabricated at Takeda Sentanchi Supercleanroom, accessible through the MEXT Nanotechnology Platform Nanofabrication Program appointed to the VLSI Design and Education Center (VDEC) and in cooperation with the Graduate School of Engineering, the University of Tokyo.

\section{References}

1 Y. Koike and S. Ueha: J. Acoust. Soc. Jpn. 61 (2005) 137 (in Japanese).

2 K. Hashiba, K. Terao, and T. Kunoh: Trans. Jpn. Soc. Mech. Eng. C 63 (1997) 3487 (in Japanese).

3 Y. Hashimoto, Y. Koike, and S. Ueha: J. Acoust. Soc. Jpn. 53 (1997) 817 (in Japanese).

4 E. Matsuo, Y. Koike, K. Nakamura, S. Ueha, and Y. Hashimoto: IEICE Tech. Rep. Ultrason. US98-79 (1998) 33 (in Japanese).

5 H. Nomura and T. Kamakura: J. Source Acoust. Soc. Jpn. 56 (2000) 805 (in Japanese).

6 T. Hatanaka, Y. Koike, K. Nakamura, S. Ueha, and Y. Hashimoto: IEICE Tech. Rep. Ultrason. 99 (1999) 15 (in Japanese).

7 S. Ueha: Revista de Acústica 33 (2002) 3.

8 M. Takasaki, D. Terada, Y. Kato, Y. Ishino, and T. Mizuno: Elsevier Phys. Procedia 3 (2010) 1059.

9 S. Chino, Y. Kato, Y. Ishino, M. Takasaki, and T. Mizuno: J. Jpn. Soc. Precis. Eng. 78 (2012) 332 (in Japanese).

10 K. Uchiage, M. Takasaki, and T. Mizuno: Proc. IEEE International Ultrasonics Symposium (2014) p. 2510.

11 S. Kuroda and Y. Hori: J. Jpn. Soc. Tribologists 21 (1976) 740 (in Japanese).

12 M. Takasaki, Y. Maruyama, and T. Mizuno: IEEE Int. Conf. Mechatronics and Automation (2007) p. 3817. 


\section{About the Authors}

Masaya Takasaki received his B.E., M.E., and Ph.D. degrees from the University of Tokyo, Tokyo, Japan, in 1996, 1998, and 2001, respectively. Since 2001, he has been with the Department of Mechanical Engineering at Saitama University, Saitama, Japan. Since 2012, he has been a Professor in the department. His current research interests include ultrasonic application for mechatronics, surface acoustic wave utilization, and design. Computer interfaces such as tactile displays are also among his interests.

Ryutaro Chida received his B.E. and M.E. degrees from Saitama University, Saitama, Japan, in 2012 and 2014, respectively.

Shota Chino received his B.E. and M.E. degrees from Saitama University, Saitama, Japan, in 2010 and 2012, respectively.

Satoshi Morishita received his BE ('08), ME ('10) and Ph.D. ('13) degrees from the Department of Electrical Engineering and Information Systems at the University of Tokyo. His research interests are CMOS integrated MEMS and related deep reactive ion etching technology for future autonomous distributed microagents.

Yuji Ishino graduated from the University of Tsukuba in 1988. Since 1988, he has been with the Department of Mechanical Engineering, Saitama University, Japan, where he is a technician. He obtained a Ph.D. degree from the Graduate School of Science and Engineering, Saitama University, Japan, in 2014. His current research interests include magnetic suspension, vibration isolation, and mass measurement under microgravity conditions.

Kota Hosaka received his BE ('11) and ME ('13) degrees from the Department of Electrical Engineering and Information Systems at the University of Tokyo. His research interests are CMOS integrated MEMS for reliable LSI testing.

Yoshio Mita has been an associate professor in the Department of Electrical Engineering and Information Systems (EEIS) since 2005, preceded by appointments as Lecturer ('01-04) and Assistant Professor ('00-01) at the University of Tokyo. He received his BE ('95), ME ('97), and Ph.D. (2000) degrees from the Department of Electrical Engineering at the University of Tokyo. Since 1995, Dr. Mita has been working on integrated intelligent micro electro mechanical systems (MEMS). While being a MEMS device and process researcher, Dr. Mita has been continuously working on multi-use MEMS fabrication platform engineering. Since 2012, he has served as a Nanotechnology Platform UTokyo VDEC site manager, where he runs a 600 $\mathrm{m}^{2}$ cleanroom environment open for over 700 users annually from over 200 research groups, including universities and companies. Dr. Mita received the Niwa Memorial Award in 2000 for his distributed MEMS controller work, and the Faculty of Engineering's Best Teaching Award in 2013. 
Takeshi Mizuno is a professor of Mechanical Engineering at Saitama University in Saitama, Japan. He received his B.E., M.E., and D.E. degrees from the University of Tokyo in 1978, 1980, and 1985, respectively. He was a research associate at the Institute of Industrial Science, the University of Tokyo, from 1980 to 1985, and an assistant professor at the Polytech University from 1985 to 1988. Since 1988, he has been with the Department of Mechanical Engineering, Saitama University. His research interests include control engineering, mechatronics, magnetic and electrostatic suspension, magnetic bearing, active vibration control, vibration isolation, dynamic vibration absorbers, micromanipulation and micro-assembly systems, and instruments for measuring mass and force. 